\title{
Evolutionary Consequences of Conditional vs. Unconditional Sharing: A Case Study in Computational Philosophy
}

\author{
Jacob S. Shaw \\ Independent researcher, United States
}

\begin{abstract}
Agent-based modeling techniques have been used by researchers in the field of computational philosophy to provide insights into a number of philosophical questions. In the study reported here, we ran a series of agent-based simulation experiments to see whether groupwide adherence to certain specific moral rules and practices might provide an evolutionary advantage. In each experiment, two groups of individuals competed with each other to harvest food. The members of one group ("sharers") then shared food with each other as specified in one of two sharing rules; members of the other group ("hoarders") never shared food. Individuals who had at least a specified amount of food survived and reproduced, while those who did not died of starvation. Changes in population size were used as a measure of evolutionary fitness. In some experiments, the sharers followed an "unconditional" sharing rule under which they were always obligated to share food. In other experiments, sharers followed a "conditional" sharing rule that made an exception when sharing could put the sharer itself at risk of starving. We found that unconditional sharing had an evolutionary advantage over hoarding when food was plentiful, but a disadvantage when food was scarce. Conditional sharing also offered an advantage over hoarding in a high-resource environment, but unlike unconditional sharing, it did not result in a disadvantage in a low-resource environment. Our observation of these evolutionarily favorable characteristics of conditional sharing provides an interesting example of the potential use of agent-based modeling to inform discussions about the evolution of morality.
\end{abstract}

Keywords: computational philosophy, agent-based modeling, sharing, evolution of morality 


\section{Introduction}

Computational philosophy is an emerging research discipline that involves the application of computational techniques and models to various areas of philosophical inquiry (Thagard, 1988; Pollock, 1995; Grim, 1996; Axelrod, 1997A; Zollman, 2005; Ruvinsky, 2007; Guarini, 2011; Grim et al. 2015; Klein et al., 2018; Thicke, 2020). In particular, some philosophical researchers have used a technique called agent-based modeling (ABM) (Epstein \& Axtell, 1996; Axelrod, 1997B; Bonabeau, 2002) to run simulations in which rules describing interactions among individuals ("agents") are explicitly coded, and observations are then made about the collective effects of these interactions on a group to which the agents belong.

In the study reported in this paper, we used ABM to investigate whether groupwide adherence to certain specific moral rules and practices might provide a survival advantage for the society that adopts them, and might thus be explainable, at least in part, in evolutionary terms. The simulation experiments we conducted relate to the ethical question of when, if ever, people have a moral obligation to share. Most people would probably agree, for example, that one should share food with (and save the life of) somebody who would otherwise starve. The situation would be more complicated, though, if sharing might cause both the giver and receiver to starve. An alternative moral precept would require one to share food unless doing so would put the giver at risk of starving. In this paper, we refer to individuals who adhere to the former moral rule as "unconditional sharers," while those who follow the latter rule are referred to as "conditional sharers." Individuals who never share are referred to as "hoarders." Our inquiry was designed to explore the relative survival advantages or disadvantages of unconditional sharing, conditional sharing, and hoarding.

In each of the simulations we conducted, a group of hoarders searches for food each day in competition with a group of either conditional or unconditional sharers. At the end of this harvesting process, the sharers (but not the hoarders) redistribute a portion of their food among themselves (in either a conditional or unconditional manner, depending on the experiment). Each individual must consume a certain amount of food each day to survive. At the end of each day, those individuals who have at least that much food reproduce, while those who have less than that amount die of starvation.

In each of our computational experiments, we monitored changes in the number of hoarders vs. sharers over time as a measure of the evolutionary impact of groupwide adherence to the two alternative sharing rules. To check for possible interactions between the overall availability of resources and the evolutionary advantages of alternative sharing rules, we performed each of these experiments under two different assumptions: a "high-resource environment" scenario, in which food is plentiful enough to support a relatively large population, and a "low-resource environment" scenario, in which food is scarce.

In our first experiment, we compared the effect of hoarding with that of unconditional sharing. We found that within a high-resource environment, the population of (unconditional) sharers tends to increase and the population of hoarders tends to decrease over time, the latter 
group often dying out entirely. But when food is scarce, the opposite occurs: the hoarder population tends to grow, and the sharer population tends to shrink.

In the second experiment, we compared the effect of hoarding with that of conditional sharing. Within a high-resource environment, the (conditional) sharer population tends to increase and the hoarders generally die out, much like the first (unconditional sharer) experiment. But when food is scarce, the two societies perform comparably well.

\section{Methods}

\subsection{Simulation structure}

All simulations are situated in a plot of land within which simulated agents walk around looking for "clumps" of food, each of which contains a given number of "units" of food. (Figure 1 shows a portion of the simulated landscape at a particular point within an actual simulation.)

Each simulation consists of 200 days. Each day is made up of five "periods": a "harvesting period," during which the agents collect food; a "sharing period," when the sharers share food among themselves; an "eating period," when all agents either eat food or die of starvation; and a "reproduction period," when agents generate offspring.

\subsubsection{Initial conditions}

All simulations begin with 150 sharers (either conditional or unconditional) and 150 hoarders. Although the number of agents in each group changes over time, agents never switch from one group to another. Each agent is initially placed at a random location, and is given a random amount of food (within a range between 225 and 350 units). Simulations always begin with 100 clumps of food, which are also assigned to random locations.

\subsubsection{Harvesting period}

In the harvesting period, the agents walk around randomly, foraging for clumps of food. If an agent is in close proximity to a clump, the clump disappears, and all of the food contained in the clump is added to the agent's own food reserve. The amount of food contained in each clump is different in the high- and low-resource conditions, but is consistent across all clumps within a given experiment. (In order to keep the total population of agents within a range in which the phenomena we are studying can be observed, the code also incorporates a foodindependent control mechanism in which agents may die of "overcrowding" if they approach each other too closely in the course of foraging.)

At the end of the day, new clumps of food grow back in random locations throughout the land to replace those eaten throughout the day. 
$3^{\text {rd }}$ International Conference on

\section{SOCIAL SCIENCES IN THE 21ST CENTURY}

Figure 1: View of the simulated environment. Conditional sharers are shown in green, hoarders are shown in red, and food clumps are shown in yellow.

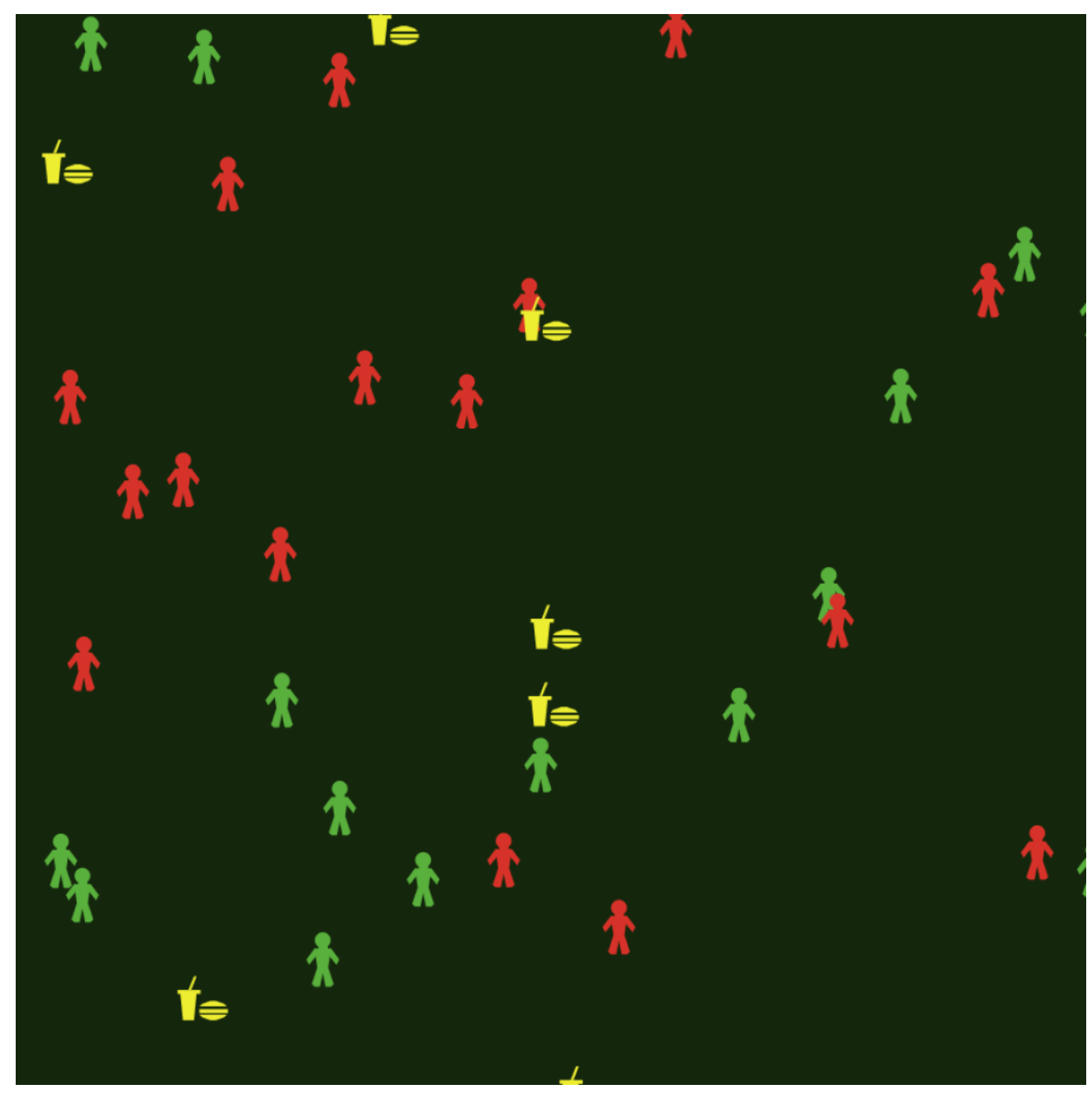




\subsubsection{Sharing period}

During the sharing period, sharers reallocate their food resources according to the sharing rule associated with the current experiment. (Hoarders never share food, so they are not affected by this reallocation process.) Specifically, in the experiment in which hoarders compete with unconditional sharers, each sharer contributes $75 \%$ of its total food reserves to a common "food bank." In the experiment in which hoarders compete with conditional sharers, each sharer contributes $75 \%$ of any food in its food reserves in excess of five times the amount of food required each day to survive. All food in the food bank is then divided equally among all living sharers.

\subsubsection{Eating period}

During the eating period, any agent that has less than 100 units of food dies of starvation, and any smaller quantity of food that agent might have disappears. If the agent does have at least 100 units of food, it "eats" 100 units of the food it has, which is subtracted from its reserves.

\subsubsection{Reproduction period}

During the reproduction period, all agents who remain alive have a $50 \%$ chance of reproducing. When an agent reproduces, another agent is created in a random location with its parent's societal affiliation (sharer or hoarder). The new agent is then given a random amount of food (again, between 225 and 350 units of food), which is newly created, and not taken from its parent's reserves.

\section{2 "High-resource" and "low-resource" conditions}

For both combinations of societies (unconditional and conditional sharers), two sets of conditions were tested: "high-resource" and "low-resource." The only parameter that differs between these two conditions is the amount of available food: 5000 units of food per clump under the former condition and 100 under the latter condition.

\subsection{Implementation}

All simulations were written and executed using NetLogo (Version 6.1.1), a programming language and integrated development environment for ABM. (Wilensky, 1999; Tisue \& Wilensky, 2004).

\section{Results}

The results of our simulation experiments are shown in Figure 2. Each graph compares changes in the number of hoarders with changes in the number of (one of the two types of) sharers over a period of 200 simulated days. The four graphs correspond to the four combinations of the two types of sharers (unconditional vs. conditional) and the two levels of available food resources (high-resource environment vs. low-resource environment). 
Figure 2: Population over 200 days of simulation, each averaged over 100 runs.

\begin{tabular}{|c|c|c|}
\hline & High-Resource Environment & Low-Resource Environment \\
\hline $\begin{array}{l}\text { Hoarders vs. } \\
\text { Unconditional } \\
\text { Sharers }\end{array}$ & $2 0 0 \longdiv { \text { CHoarders—Unconditional sharers } }$ & ${ }^{400}$ \\
\hline $\begin{array}{l}\text { Hoarders vs. } \\
\text { Conditional } \\
\text { Sharers }\end{array}$ & Con & $300 \overline{ }$ \\
\hline
\end{tabular}

A total of 100 simulations were run for each of these four combinations. The data point shown in Figure 2 for a given day is the mean value of the population on that day, averaged over these 100 simulation runs. Although these average values changed over time in a reproducible, relatively smooth way, the timing of various significant events (for example, major population changes, or arrival at a stable equilibrium range) differed significantly from one simulation run to another. Moreover, the results of individual simulations occasionally ran contrary to the averaged results shown above - in the low-resource environment, for example, the hoarders almost always wound up outnumbering the sharers, but in a small minority of cases, the unconditional sharer population surpassed that of the hoarders.

As shown in the top two graphs of Figure 2, we found that unconditional sharing offered an advantage over hoarding when food was plentiful, but a disadvantage when food was scarce. As seen in the bottom two graphs of Figure 2, conditional sharing also offered an advantage over hoarding in a high-resource environment, but unlike unconditional sharing, it did not result in a disadvantage in a low-resource environment.

The main results of these four simulation experiments are summarized in Table 1. 
Table 1: Summary of key results.

\begin{tabular}{|c|c|c|}
\hline & High-Resource Environment & Low-Resource Environment \\
\hline $\begin{array}{c}\text { Hoarders vs. } \\
\text { Unconditional } \\
\text { sharers }\end{array}$ & Sharers dominate & Hoarders dominate \\
\hline $\begin{array}{c}\text { Hoarders vs. } \\
\text { Conditional } \\
\text { sharers }\end{array}$ & Sharers dominate & $\begin{array}{c}\text { Hoarders \& sharers } \\
\text { perform comparably }\end{array}$ \\
\hline
\end{tabular}

\section{Conclusion}

In the study reported in this paper, we used agent-based modeling techniques to evaluate the extent to which the evolutionary fitness of a society might be affected by its adherence to different moral rules and practices related to the sharing of resources. In particular, we ran (many repetitions of) a series of agent-based simulation experiments in which a group of individuals who share resources in accordance with one of two sharing rules (conditional sharing or unconditional sharing) competed for resources with a group of individuals who do not share resources. We conducted each of these experiments under conditions of both high and low levels of resource availability to see whether this affected the relative advantages provided by the sharing rules. Population size was used as a measure of evolutionary fitness.

We found that unconditional sharing offered an evolutionary advantage over hoarding in a high-resource environment, but a disadvantage in a low-resource environment. Conditional sharing also offered an advantage over hoarding in a high-resource environment, but unlike unconditional sharing, it did not result in a disadvantage in a low-resource environment.

Our findings might lend an evolutionary perspective to rules incorporated in certain religious doctrines and economic policies. In Islam, believers are religiously obligated to contribute a portion of their wealth to others who are less fortunate-but only if their wealth exceeds a certain level, called nisab. (It has been suggested that nisab was likely an approximation of how much wealth a family of six would need to feed themselves for approximately one year (Jawaid, 2020).) In Britain, the belief that people with very limited resources should be fully exempt from paying taxes was "a basic principle of taxation since the age of Adam Smith,” according to John Veit-Wilson (Veit-Wilson, 1999). 
It seems possible that agent-based simulation methods like those used in this study could help provide evolutionary context for other problems in moral philosophy.

\section{References}

Axelrod, R. (1997A). The dissemination of culture: A model with local convergence and global polarization. The Journal of Conflict Resolution, 41(2), 203-226.

Axelrod, R. (1997B). The Complexity of Cooperation: Agent-Based Models of Competition and Collaboration. Princeton, NJ, Princeton University Press.

Bonabeau, E. (2002). Agent-based modeling: Methods and techniques for simulating human systems. Proceedings of the National Academy of Sciences, 99(Supplement 3), 7280-7287.

Epstein, J. M. \& Axtell, R. L. (1996). Growing Artificial Societies: Social Science from the Bottom Up. Cambridge, MA, MIT Press.

Guarini, M. (2011). Computational neural modeling and the philosophy of ethics: Reflections on the particularism-generalism debate. In M. Anderson \& S. Anderson (Eds.) Machine Ethics (pp. 316-334). Cambridge: Cambridge University Press.

Grim, P. (1996). Spatialization and greater generosity in the stochastic Prisoner's Dilemma. Biosystems, 37(1-2), 3-17.

Grim, P., Singer, D., Reade, C., and Fisher, S. (2015). Germs, genes, and memes: Function and fitness dynamics on information networks. Philosophy of Science, 82, 219-243.

Jawaid, M. (2020). The Basis of Nisab. from https://www.academia.edu/42609061/The_Basis_of_Nisab

Klein, D., Marx, J., and Fischbach, K., (2018). Agent-based modeling in social science, history, and philosophy: An introduction. Historical Social Research, 43, 7-27.

Pollock, J. L. (1995). Cognitive Carpentry: A Blueprint for How to Build a Person. MIT Press.

Ruvinsky, A. I. (2007). Computational Ethics. In M. Quigley (Eds.), Encyclopedia of Information Ethics and Security (pp. 76-82). IGI Global.

Thagard, P. (1988). Computational Philosophy of Science. Cambridge, MA: MIT Press.

Thicke, M. (2020). Evaluating formal models of science. Journal for General Philosophy of Science, 51, 315-335.

Tisue, S. and Wilensky, U. (2004). NetLogo: A simple environment for modeling complexity. International Conference on Complex Systems, 21, 16-21. 
Veit-Wilson, J. (1999). The tax threshold: policy, principles, and poverty. Twentieth Century British History, 10(2), 218-234.

Wilensky, U. (1999). NetLogo (Version 6.1.1) [Computer software]. Evanston, IL: Center for Connected Learning and Computer-Based Modeling, Northwestern University. Retrieved from http://ccl.northwestern.edu/netlogo/

Williams, Bernard A. O. (1973). Egoism and altruism. In Problems of the Self: Philosophical Papers 1956-1972. Cambridge, Cambridge University Press.

Zollman, K. (2005). Talking to neighbors: The evolution of regional meaning. Philosophy of Science, 72(1), 69-85. 